\title{
Design of the ATLAS New Small Wheel Gas Distribution System for the Micromegas Detector Modules
}

\section{E. N. Gazis ${ }^{* a}$, On behalf of the ATLAS NSW Collaboration}

${ }^{a}$ National Technical University of Athens, Greece

E-mail: evangelos.gazisecern.ch

In this work we present and describe the methodology, the relative calculations and simulations accordingly to achieve the appropriate gas flow rates ensuring a uniform gas distribution among the same type of New Small Wheel (NSW) Micromegas modules. The majority of the components used are in large multiplicity so space saving criteria is taking into account and simplicity on the performance with respect to the total cost as well. An appropriate simulation program has been developed for studying the overall gas system determining the gauge pressure, flow rate in the critical points and branches, respectively. Moreover, an overall prototype configuration, implemented at the NTUA laboratory and based on the Lock-in Amplifier technique to be used in conjunction with the gas leak test via the FRL method is presented. The obtained performances, by means of sensitivity and $\mathrm{S} / \mathrm{N}$ ratio improvement, are also discussed.

PACS: ATLAS, NSW, Micromegas, gas system

Fourth Annual Large Hadron Collider Physics

13-18 June 2016

Lund, Sweden

* Speaker. 


\section{Introduction}

Large scale resistive Micromegas (MM) detectors will be employed for the first time in highenergy physics experiments for the Phase-I upgrade of the ATLAS Muon Spectrometer at LHC/CERN [■, 四]. The current innermost stations of the muon endcap system, the Small Wheel, will be upgraded in 2019 to retain the good precision tracking and trigger capabilities in the high background environment expected with the upcoming luminosity and energy increase of the LHC. In particular, the innermost station of the endcap region will be replaced by a new system (New Small Wheel) [B]. MicroMegas (Micro-Mesh Gaseous Structure MM) [4] detectors have been chosen as the main tracking chambers and, at the same time, will also contribute to the trigger, which will be provided mainly by the small-strip Thin Gap Chambers (sTGC). The upgrade is to retain the good precision tracking and trigger capabilities in the high background environment expected with the upcoming luminosity and energy increase of the LHC.

\section{The New Small Wheel (NSW) for the ATLAS upgrade}

The NSW upgrade is motivated primarily by the high background rate that is expected at a Luminosity in the range $2-7 \times 10^{34} \mathrm{~cm}^{-2} \mathrm{~s}^{-1}$ during LHC Run-3 and HL-LHC. NSW will replace with fast, high rate, precision detectors for high rate up to $20 \mathrm{kHz} / \mathrm{cm}^{2}$ with resolution less than $100 \mu \mathrm{m}$ per plane. NSW will provide improved trigger for forward muons present L1 trigger and improved tracking: New (fast) precision tracker in NSW that works up to ultimate luminosity $7 \times 10^{34} \mathrm{~cm}^{-2} \mathrm{~s}^{-1}$, with some safety margin and kill the fake triggers by requiring high quality $\left(\sigma_{\theta} \sim\right.$ $1 \mathrm{mrad}$ ) pointing segments in NSW. Furthermore, as shown in Fig. W a significant improvement in the level-1 trigger rate as a function of muon $p_{T}$ will be achieved by using the NSW detector [可].

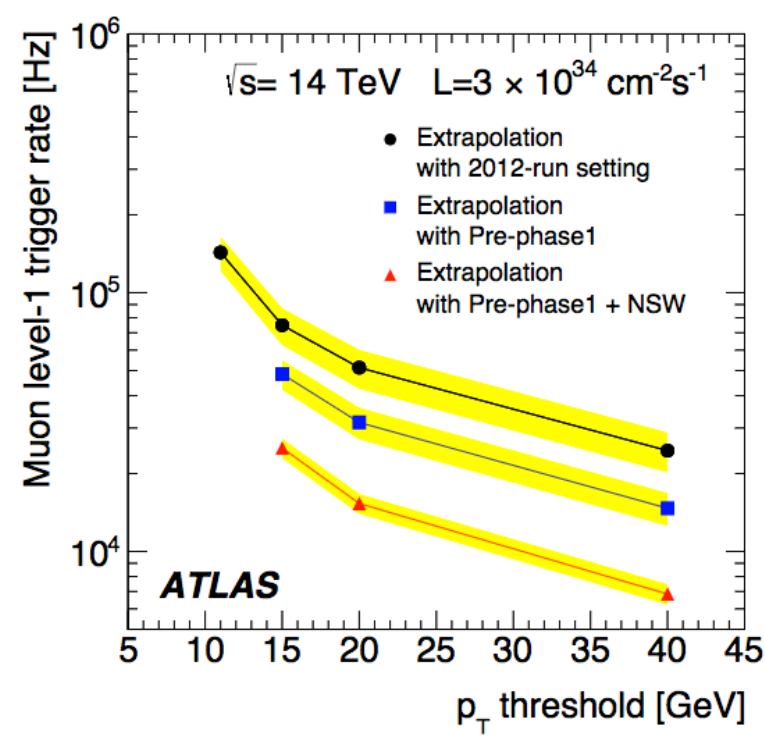

Figure 1: Plot showing the level-1 trigger level as a function of transverse momentum, $p_{T}$, threshold for the existing SW and the NSW [[]]. 


\section{The Micromegas gas distribution system}

The NSW consists of 16 detector planes in two multilayers. Each multilayer comprises four small-strip TGC (sTGC) and four Micromegas (MM) detector planes. The MM detectors have exceptional precision tracking capabilities due to their small gap (5mm) and strip pitch $(0.5 \mathrm{~mm})$. They are arranged in large and small sectors. The dimensions of the sectors are chosen such that approximately the same azimuthal overlap of the active areas as in the current Small Wheel is achieved. Each sector comprises of eight MM detection layers, grouped into two quadruplets of four layers each (hence quadruplets), separated by a $40 \mathrm{~mm}$ thick spacer. Figure $\square$ shows schematically the arrangement of the detectors in a sector. Each quadruplet contains four active layers, grouped into two pairs, as illustrated in Fig. []; both have taken from the Ref. [3]].
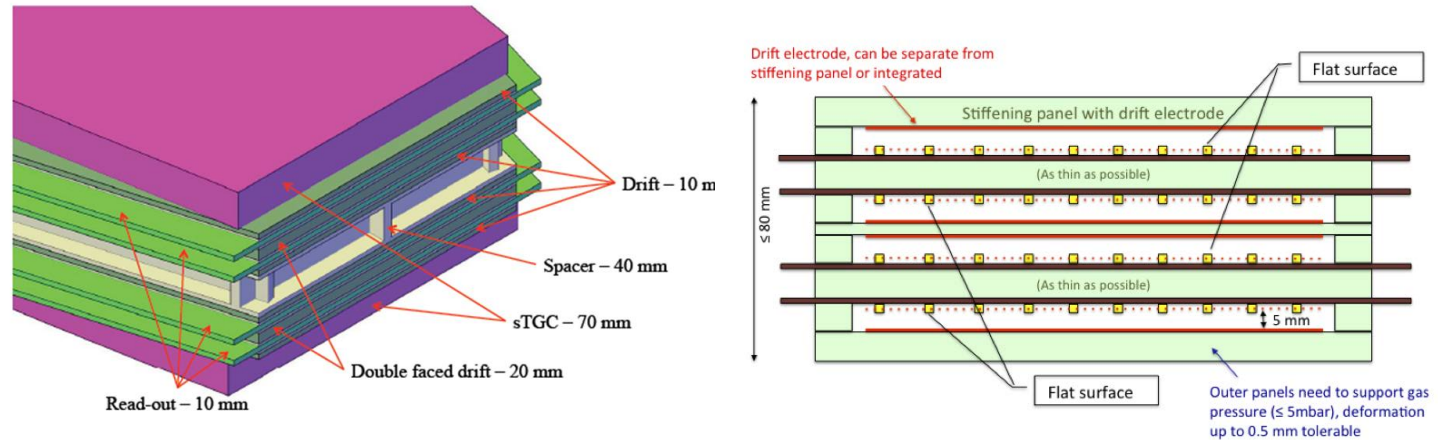

Figure 2: Arrangement of the detectors in a sector [3]].
Figure 3: Arrangement of the detectors in a quadruplet [B]].

The MM detectors will operate with gas mixture $\mathrm{Ar} / \mathrm{CO}_{2}$ (93:7) at atmospheric pressure. A single gas distribution system for them has been properly designed [ [6]. The calculations and simulations guide to achieve the sustainable gas flow rates ensuring a uniform gas distribution among the same type of modules. The majority of the components used are in large multiplicity so space saving criteria is taking into account and simplicity on the performance with respect to the total cost as well.

The NSW gas setup, includes 16 channels serving two wedges, but not of the same sector in order to retain redundancy. The configuration is shown in Fig. 团 for the two MM types covering the whole wheel, but showing only IP-side wedges [6].

A high importance objective of our design was the study of the functionality of the whole distribution system. The particular pressures in inlets and outlets of the MM layers and as well as the gas flow rate though them should be the crucial quantities in the real-final system. The system study was divided in two parts: in the first approach, the goal was to determine the pressure/flow rate relationship of the different gas components, or in other words, their local resistance due to minor losses by the help either theoretical models or individual simulations using COMSOL Multiphysics and ANSYS CFX [0, [0]]. In the second study the gas distribution system by using the Pipe Flow package [ [ $]$ ] was simulated. The associated components have been included in the 


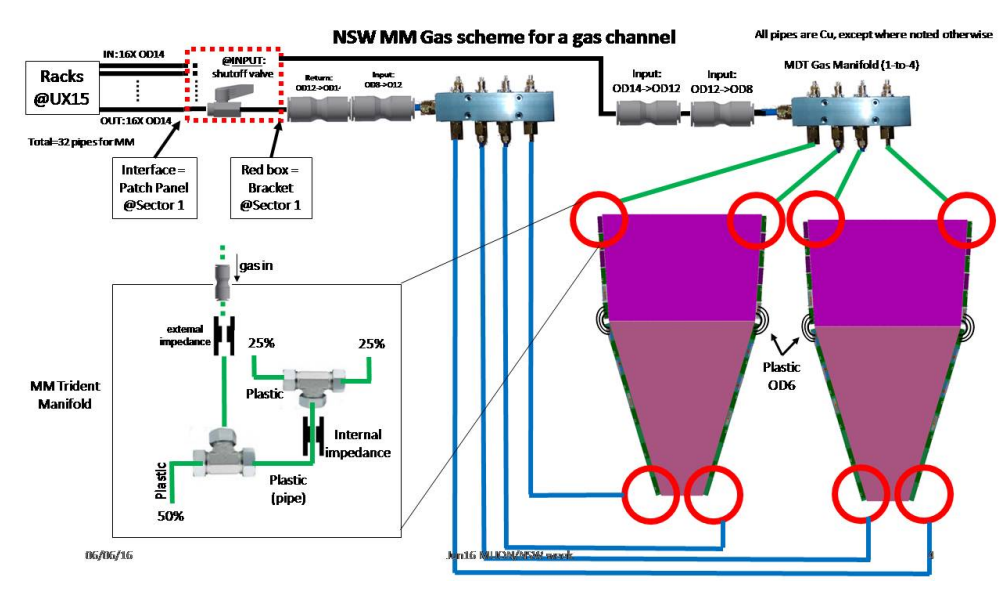

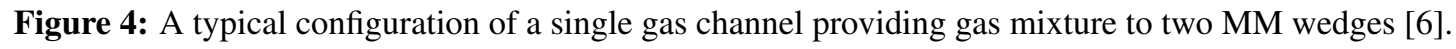

simulation by means of their equivalent resistance (equal to their local resistance found in the first study). For the impedances the functional curve specifying a number of points (pressure drop versus gas flow rate) was introduced. The actual routing of the pipes and their associated lengths were also introduced according to two alternative solutions: a) the baseline routing by which the pipes return to the gas rack by the inner rim, $b$ ) alternative routing by which the pipes return to the gas rack via radial paths between the wedges.

Different studies we have done concern the gas mixture propagation through the micromesh structure to avoid "inefficient" areas into the chamber for its best performance. The study is based on the following parameters: a) nominal flow rate at $5 \mathrm{NL} / \mathrm{h}$ on each buffer zone inlet, b) number of injection holes must be optimized c) empirical ratio between the buffers zone size and the hole diameter (dbz/dhole $\sim 10$ ) is used. In Fig. $\$$ a typical result of the simulation is presented where a uniform flow of the gas mixture is indicated by the red color while by glue color the initial stage of air is indicated [目].

\section{Gas leak tightness test methods}

According to the NSW requirements a general rule for the leak rates of the modules have specified that the leak rate has to be $10^{-5} \times V$ per minute, where $V$ is the volume of the module. The obtained limits differ because of the different volumes. However, the pressure (gauge pressure) of the main test schedule must be specified. In addition, when a quadruplet (four modules in backto-back orientation) is created, the gas mixture can flow among the four modules. This lead to the necessity for testing the complete quadruplet and not a single Modules alone.

A Gas Leak monitored of the NSW MicroMegas quadruplets strategy, in the framework of quality checking-quality assurance, has been selected combining a conventional one based on the Pressure Decay Rate (PDR) and a novel one, the Flow Rate Loss (FRL) [प]. An overall prototype configuration, implemented at the NTUA laboratory and based on the Lock-in Amplifier technique to be used in conjunction with the gas leak test via the FRL method. In Fig. 6 typical flow rates measured in input and output are presented using the calibrated needle $31 \mathrm{G}$ [四]. 


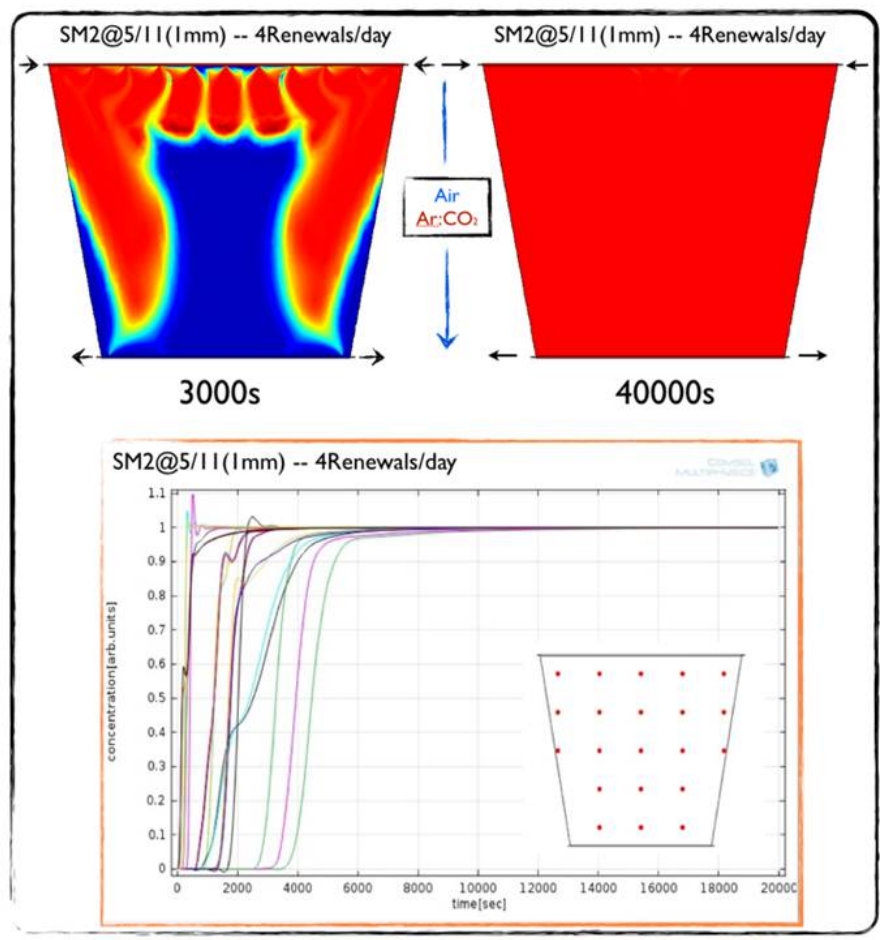

Figure 5: Simulation study of the gas flow behavior of the SM2 chamber (upper part). The time evolution of the gas mixture concentration inside the MM module in different test points (down part) [6].

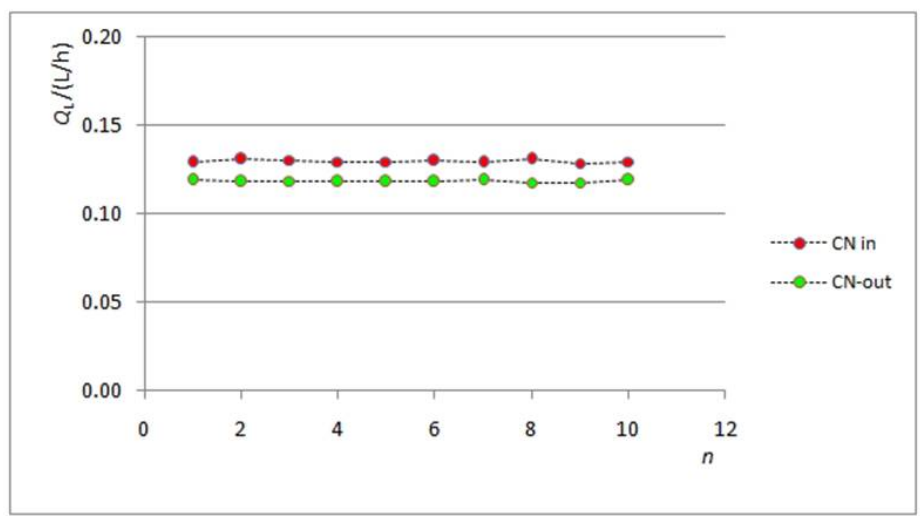

Figure 6: The flow rates measured in the input and output with the calibrated needle $31 \mathrm{G}-\mathrm{CN} 1$ connected in the stream line [ $[\mathbf{D}]$. 


\section{Conclusions}

In this work the Gas Distribution System to provide the required gas flow rate into the Micromegas Detectors for the upgrade of the ATLAS experiment in the NSW project is described. The associated components (impedances, maniflods, etc) and their behavior in the system have been studied by simulations and experimental laboratory tests.

Two combined gas leak methods for the NSW MicroMegas quadruplets QA/QC, the pressure decay rate (PDR) and the novel proposed flow rate loss (FRL), have been implemented in a unified prototype setup. The calibration of both methods introduced the technique of the Leak Branches based on specific medical needles. The gas leak prototype setup, as first-level PDR/FRL configuration, will be used during the Module-0 Micromegas tests and during the QA/QC of the production modules.

\section{References}

[1] ATLAS Collaboration, Journal of Instrumentation 3 (2008) S08003.

[2] LHC Collaboration, Journal of Instrumentation 3 (2008) S08001.

[3] ATLAS Collaboration, ATLAS New Small Wheel TDR, CERN-LHCC-2013-006 ATLAS-TDR-020-2013.

[4] Y. Giomataris, P. Rebourgeard, J.P. Robert, G. Charpak, Physics Research Section A 376 (1996) 29, T. Alexopoulos, et al., Instr. Meth. A 640, 110-118, (2011).

[5] ATLAS Collaboration. Technical Design Report for the Phase-I Upgrade of the ATLAS TDAQ System. CERN-LHCC-2013-018. ATLAS-TDR-023. Geneva: CERN, Sept. 2013.

[6] T. Alexopoulos, et al., Design of NSW Gas Distribution System for Micromegas Detectors, ATLAS NOTE, to be published.

[7] T. Alexopoulos, et al., Gas Leak Test Prototype Setup for the NSW Micromegas Multiplets: 4 Implementation and Calibration Technique, ATLAS NOTE, to be published.

[8] 'PipeFlow', http://www.pipeflow.co.uk/.

[9] 'Comsol multiphysics', http://www.comsol.com/comsol-multiphysics.

[10] 'ANSYS CFX', http://www.ansys.com/Products/Simulation+Technology/Fluid + Dynamics/Fluid+Dynamics + Products/ANSYS + CFX. 